\title{
Generation of Kidney from Pluripotent Stem Cells via Blastocyst Complementation
}

\author{
Jo-ichi Usui, ${ }^{*}$ Toshihiro Kobayashi, ${ }^{\star \dagger}$ \\ Tomoyuki Yamaguchi, ${ }^{* \dagger}$ A.S. Knisely, ${ }^{\ddagger}$ \\ Ryuichi Nishinakamura, ${ }^{\S}$ and \\ Hiromitsu Nakauchi ${ }^{\star \dagger}$ \\ From the Division of Stem Cell Therapy,* Center for Stem Cell \\ Biology and Regenerative Medicine, Institute of Medical Science, \\ University of Tokyo, Tokyo, Japan; the Japan Science Technology \\ Agency, ${ }^{\dagger}$ ERATO, Nakauchi Stem Cell and Organ Regeneration \\ Project, Tokyo, Japan; the Institute of Liver Studies, ${ }^{\ddagger}$ King's \\ College Hospital, London, United Kingdom; and the Department \\ of Kidney Development,${ }^{\S}$ Institute of Molecular Embryology and \\ Genetics, Kumamoto University, Kumamoto, Japan
}

Because a shortage of donor organs has been a major obstacle to the expansion of organ transplantation programs, the generation of transplantable organs is among the ultimate goals of regenerative medicine. However, the complex cellular interactions among and within tissues that are required for organogenesis are difficult to recapitulate in vitro. As an alternative, we used blastocyst complementation to generate pluripotent stem cell (PSC)-derived donor organs in vivo. We hypothesized that if we injected PSCs into blastocysts obtained from mutant mice in which the development of a certain organ was precluded by genetic manipulation, thereby leaving a niche for organ development, the PSC-derived cells would developmentally compensate for the defect and form the missing organ. In our previous work, we showed proof-of-principle findings of pancreas generation by injection of PSCs into pancreas-deficient $P d x 1^{-1-}$ mouse blastocysts. In this study, we have extended this technique to kidney generation using Sall $^{-/-}$ mouse blastocysts. As a result, the defective cells were totally replaced, and the kidneys were entirely formed by the injected mouse PSC-derived cells, except for structures not under the influence of Sall1 expression (ie, collecting ducts and microvasculature). These findings indicate that blastocyst complementation can be extended to generate PSC-derived kidneys. This system may therefore provide novel insights into renal organogenesis. (Am J Pathol 2012, 180:2417-2426; bttp:// dx.doi.org/10.1016/j.ajpath.2012.03.007)
At present, most patients with end-stage renal failure are treated by dialysis. Some patients eventually undergo kidney transplantation, but this option is limited by a shortage of donor organs. Therefore, the number of patients undergoing dialysis continues to grow, with complications, poor quality of life, and increasing medical costs. Moreover, the shortage of donor organs has led to other social problems, such as organ trafficking, transplant tourism, and transplant commercialism. ${ }^{1}$ Under these circumstances, to transplant donor organs derived from pluripotent stem cells would be a much welcomed alternative. Induced-PSC (iPSC) technology ${ }^{2,3}$ has recently enabled the generation of individual- or patientderived PSCs, with studies of disease-targeting stem cell replacement therapy. However, the generation of an organ from iPSCs is considered impractical because it remains difficult to replicate in vitro the complex interactions among cells and tissues during organogenesis. To overcome this obstacle, we attempted to generate organs in vivo using the blastocyst complementation technique originally reported by Chen et $\mathrm{al}^{4}$ in analyses of genes involved in lymphocyte development. We reported the successful application of this technique to generate PSC-derived mouse and rat pancreas in the $P d \times 1^{-1-}$ mouse. ${ }^{5}$ In the PSC-generated pancreas, defective cells were totally replaced, and the pancreas was formed almost entirely by the injected mouse and rat PSC-derived cells. The mouse and rat PSC-derived pancreas produced a variety of hormones, including insulin, and the transplantation of PSC-derived pancreas islets improved hyperglycemia in a diabetic mouse model. The premise driving this work is that a niche for organogenesis can be created in postblastocyst mutant mouse embryos that are genetically precluded from developing a particular or-

Supported by grants from the Japan Science Technology Agency (Ministry of Education, Culture, Sport, Science, and Technology, Japan).

Accepted for publication March 1, 2012.

J.U. and T.K. contributed equally to this work.

Current address of J.U., Department of Nephrology, Faculty of Medicine, University of Tsukuba, Tsukuba, Japan.

Address reprint requests to Hiromitsu Nakauchi, M.D., Ph.D., Division of Stem Cell Therapy, Center for Stem Cell Biology and Regenerative Medicine, Institute of Medical Science, University of Tokyo, 4-6-1 Shirokanedai, Minatoku, Tokyo 108-8639, Japan. E-mail: nakauchi@ims.u-tokyo.ac.jp. 
gan. Injected PSC-derived cells will colonize this developmental niche and compensate for the developmental defect to form a donor-induced organ in vivo.

Here we report our extended application of blastocyst complementation to generate kidneys from PSCs using Sall $1^{-1-}$ mice. Sall1 is the mammalian orthologue of the Drosophila region-specific homeotic gene spalt (sal). Sall1 expression in the organism is restricted temporally to the period of nephrogenesis and disappears thereafter. Sall1 expression in the embryonic and newborn kidney is restricted histologically to metanephric mesenchyme and renal stroma. In embryonic kidney development, Sall 1 is essential for ureteric bud attraction toward the mesenchyme. Mice deficient in Sall1 therefore die soon after birth because of kidney agenesis or severe dysgenesis, whereas structures formed independently of Sall1 such as the ureters and bladder are present. ${ }^{6}$ This study was performed to establish whether Sall1 $1^{-1-}$ blastocysts offer a developmental niche for kidney tissue and could therefore be used to generate PSC-derived kidneys via blastocyst complementation.

\section{Materials and Methods}

\section{Animals}

C57BL6/NCrSlc, BDF1, and ICR mice were purchased from SLC Japan (Shizuoka, Japan). 129/OlaHsd (129/ Ola) mice were purchased from Jackson Laboratory (Bar Harbor, ME). Sall1 mutant-EGFP knock-in heterozygous mice were used for the production of ESC-derived chimeras $^{7}$ or Sall1 knock-out heterozygous mice were used for the production of iPSC-derived chimeras. ${ }^{6}$ They were crossed with C57BL/6 or BDF1 strain mice. In the Sall1 mutant-EGFP knock-in heterozygous or homozygous mouse, EGFP fluorescence was limited to Sall1-expressing cells. All experiments were performed in accordance with the animal care and use guidelines of the Institute of Medical Science at the University of Tokyo.

\section{Culture of Embryonic Stem Cells/iPSCs}

Undifferentiated mouse embryonic stem cells (ESCs: EB3-DsRed) were maintained on gelatin-coated dishes without feeder cells in Glasgow's modified Eagle's medium (GMEM; Sigma-Aldrich, St. Louis, MO) supplemented with $10 \%$ fetal bovine serum (FBS; Nichirei Bioscience, Tokyo, Japan), $0.1 \mathrm{mmol} / \mathrm{L}$ 2-mercaptoethanol (Invitrogen, San Diego, CA), 0.1 mmol/L nonessential amino acids (Invitrogen), $1 \mathrm{mmol} / \mathrm{L}$ sodium pyruvate (Invitrogen), 1\% L-Glutamine Penicillin Streptomycin (Sigma-Aldrich), and $1000 \mathrm{U} / \mathrm{mL}$ of leukemia inhibitory factor (LIF; Millipore, Bedford, MA). The EB3-DsRed cells, gifts from Dr. Hitoshi Niwa (CDB RIKEN), were derived from EB3 ES cells and carried the DsRed.T4 gene under the control of the CAG expression unit. The EB3 cells were a subline derived from E14tg2a ES cells ${ }^{8}$ and were generated by targeted integration of the Oct-3/4-IRES-BSD-pA vector into the Oct3/4 allele. $^{9}$

Undifferentiated induced pluripotent stem cells (iPSCs: GT3.2) from mice were maintained on mitomycin-C-treated mouse embryonic fibroblast (MEF) cells in Dulbecco's modified Eagle's medium (DMEM; Invitrogen) supplemented with $15 \%$ knockout serum replacement (KSR; Invitrogen), $0.1 \mathrm{mmol} / \mathrm{L}$ 2-mercaptoethanol, $0.1 \mathrm{mmol} / \mathrm{L}$ nonessential amino acids, $1 \mathrm{mmol} / \mathrm{L}$ HEPES buffer solution (Invitrogen), $1 \%$ L-Glutamine Penicillin Streptomycin, and $1000 \mathrm{U} / \mathrm{mL}$ of LIF. The GT3.2 cells were generated from tail-tip fibroblasts of a male GFP transgenic mouse, kindly provided by Dr. Masaru Okabe (Osaka University) by the introduction of three factors (Klf4, Sox2, Oct3/4) in retroviral vectors. Viruses were prepared as described. ${ }^{10}$ That GT3.2 cells ubiquitously express EGFP under the control of the CAG expression unit had been established. ${ }^{5}$

\section{Embryo Manipulation}

Preparation of Sall1 heterozygous intercrossing embryos was performed as described. ${ }^{11}$ In brief, eight cell/morulastage embryos were collected in M2 medium (Millipore) from the oviducts and uteri of 2.5-day postcoital (dpc) Sall1 heterozygous mice. These embryos were transferred into KSOM-AA medium (Millipore) droplets and cultured for 24 hours until development to the blastocyst stage.

For micromanipulation of the embryos, blastocysts were transferred into M2 medium droplets and the ESCs/ iPSCs were trypsinized and suspended in the culture medium droplets. For each blastocyst, after careful drilling of the zona pellucida and trophectoderm under microscopy using a piezo-driven micromanipulator (Prime Tech, Tokyo, Japan), 10 to 15 ESCs/iPSCs were introduced into the blastocyst cavity near the inner cell mass. DsRed-marked ESCs were injected into blastocysts obtained by intercrossing of Sall $1^{+/ E G F P}$ mice; EGFPmarked iPSCs were injected into blastocysts obtained by intercrossing of Sall $1^{+/-}$mice. After injection, the embryos were cultured in KSOM-AA medium for 1 to 2 hours and were thereafter transferred into the uteri of $2.5-\mathrm{dpc}$ pseudopregnant recipient ICR mice.

\section{Flow Cytometry and Genotyping Analysis of ESC-Derived Chimeras}

To identify the genotypes of neonatal Sall1-EGFP knock-in mice, EGFP-positive cells from the brain and kidney were sorted by fluorescence-activated cell sorting and genotyped by genomic PCR. The analysis and sorting were performed by Mo-flo (Beckman Coulter, Fullerton, CA) or using a FACS Vantage SE system (BD Bioscience Pharmingen, Franklin Lakes, NJ). DNA was extracted from collected cells using a QIAamp DNA Mini Kit (Qiagen, Germantown, MD) and was used to confirm mouse genotypes. The PCR primers used for the amplification of the wild-type Sall 1 allele and the mutated allele were as follows: 5'-AGCTAAAGCTGCCAGAGTGC-3', 5'-CAACTTGCGATTGCCATAAA-3', and 5'-GCGTTGGCTACCCGTGATAT-3' (288 bp for the wild-type Sall1 allele and 350 bp for the mutated allele). ${ }^{7}$ Nested PCR primers were as follows: 5'-AGAATGTCGCCCGAGGTTG-3', 5'-TACAGCAAGCTAGGAGCAC-3', and 5'- AAGAGCTTGGCGGCGAATG-3' (237 bp for the wild-type Sall1 allele 
and $302 \mathrm{bp}$ for the mutated allele). PCR amplification was performed as described elsewhere. ${ }^{6,7}$

\section{Genotyping of iPSC-Derived Chimeras Using a Single EGFP-KSL Cell-Derived Colony or Splenocytes}

To identify the genotypes of neonatal mice among the Sall1 knock-out mice, single hematopoietic stem cells (HSCs) were assayed. Bone marrow cells were obtained from neonatal mice and suspended in PBS containing 3\% FCS. Cells were stained with phycoerythrin (PE)-conjugated anti-Sca-1 monoclonal antibody (mAb; BD Bioscience Pharmingen), allophycocyanin (APC)-conjugated anti-C-Kit $\mathrm{mAb}$, and a biotinylated anti-lineage antibody cocktail containing anti-Gr-1, anti-Mac-1, anti-B220, antiCD4, anti-CD8, and anti-Ter-119 mAbs (e-Bioscience, Kyoto, Japan). The biotinylated antibodies were developed with APC-Cy7-conjugated streptavidin (SA: e-Bioscience). Single EGFP-negative KSL cells were collected in individual wells of round-bottomed, 96-well plates and cultured 10 to 14 days under conditions like those described, ${ }^{5}$ but with one modification (erythropoietin was included in the culture medium instead of G-CSF, IL-6, and IL-11). EGFP-negative splenocytes were also prepared from the same mice and were collected by Mo-flo. DNA was extracted from colony-forming cells derived from single EGFP-KSL cells or splenocytes. The PCR primers used to amplify the neomycin-resistance (neo') gene inserted in the Sall1 locus were: neor locus, forward 5'-AAGGGACTGGCTGCTATTGG-3' and reverse 5'ATATCACGGGTAGCCAACGC-3' (420 bp); Sall1 wildtype locus, forward 5'-GTACACGTTTCTCCTCAGGAC-3' and reverse 5' - TCTCCAGTGTGAGTTCTCTCG-3' (200 bp). ${ }^{6}$ PCR amplification was performed as described above.

\section{Histological Analysis}

Kidneys were fixed with $4 \%$ paraformaldehyde and embedded in paraffin or, for frozen sections, in Optimal Cutting Temperature compound (Sakura Finetek, Japan). Paraffin sections were deparaffinized with xylene and hydrated with graded ethanols. A microwave oven was used for antigen retrieval. Paraffin sections were stained with hematoxylin and eosin (H\&E) or periodic acidmethenamine silver (PAM) for light microscopy. Frozen sections were stained immunohistochemically. Briefly, each section was incubated with primary antibody overnight at $4^{\circ} \mathrm{C}$ and with secondary antibody for 1 hour at room temperature. Primary antibodies were anti-EGFP polyclonal antibody ( $\mathrm{pAb}$ ) for EGFP signal amplification (rabbit IgG, 1:500 dilution; Invitrogen), anti-DsRed pAb (rabbit IgG, 1:50 dilution; Clontech Laboratories, Mountain View, CA), anti-platelet endothelial cell adhesion molecule-1 (Pecam1) (mAb; rat IgG, 1:100 dilution; BD Bioscience Pharmingen), anti-aquaporin 1 (Aqp1) (pAb; rabbit IgG, 1:200 dilution; Chemicon International, Temecula, CA), anti-Liv2 (mAb; rat IgG, 1:200 dilution; Medical \& Biological Laboratories, Nagoya, Japan), and antineuron class III $\beta$-tubulin (Tuj1) (mAb; rabbit IgG, 1:100 dilution; Covance, Emeryville, CA). Rhodamine- and biotin-labeled Dolichos biflorus agglutinin (DBA)-lectin, Lotus tetragonolobus (LT)-lectin, and peanut agglutinin (PNA)lectin (Vector Laboratories, Burlingame, CA) staining was also performed. The secondary antibodies and fluorescence-conjugated streptavidin used for immunofluorescence studies were as follows: Alexa488-conjugated, Alexa546-conjugated, and Alexa647-conjugated goat antirabbit IgG (Invitrogen), Alexa546-conjugated and Alexa647conjugated goat anti-rat IgG (Invitrogen), and Alexa546-conjugated and Alexa647-conjugated streptavidin (Invitrogen). After treatment with the appropriate antibodies, the sections were mounted with Vectashield mounting medium (Vector Laboratories) containing DAPI for nuclear staining and were observed by fluorescence microscopy or confocal laser scanning microscopy.

For ultrastructural analysis of glomeruli, chimeric kidneys were cut into tissue blocks (approximately $0.5 \mathrm{~mm}^{3}$ ) and immersed in $2.5 \%$ phosphate-buffered glutaraldehyde overnight at $4^{\circ} \mathrm{C}$. The tissue blocks were postfixed in $1 \%$ osmium tetroxide for 2 hours at $4^{\circ} \mathrm{C}$, dehydrated in graded ethanols, and embedded in epoxy resin (Poly/ Bed 812 Embedding Media, Polysciences, Warrington, $P A)$. Ultra-thin sections cut on a Leica Ultracut S microtome (Leica, Vienna, Austria) were stained with uranyl acetate and lead citrate. Sections were observed by transmission electron microscopy (TEM) at $75 \mathrm{kV}$ using a H-7000 instrument (Hitachi, Tokyo, Japan).

\section{Results}

\section{Generation of Kidney from Embryonic Stem Cells}

We conducted blastocyst complementation using ESC and Sall 1 EGFP/EGFP blastocysts that would provide a niche for kidney development. As a result of blastocyst complementation (Figure 1, A and B), kidneys in Sall $1^{+/+}$ chimeric neonatal mice were red (data not shown). Kidneys in Sall $1^{+/ E G F P}$ chimeric mice were yellow, reflecting a merger of host-derived EGFP-expressing cells and injected DsRed-marked ESC-derived cells, as in wholebody chimerism (Sall1+/EGFP in Figure 1B). On flow cytometry analysis, the brains and kidneys of Sall $1^{+/+}$ chimeric mice contained EGFP-negative and DsRed-expressing cells (Figure 2, C and D). The brains and kidneys of Sall1 $1^{+/ E G F P}$ chimeric mice contained both EGFP-expressing and DsRed-negative cells and EGFPnegative and DsRed-expressing cells (Figure 2, E and F). The genotype of each fraction of sorted cells was confirmed by PCR (data not shown). The brains and kidneys of Sall $1^{+/ E G F P}$ mice lacking ESC chimerism contained EGFP-expressing and DsRed-negative cells (Figure 2, A and B). The kidneys of Sall $1^{+/ E G F P}$ mice intrinsically contained a few EGFP-expressing and DsRed-expressing cells (Figure 2B). In contrast, the kidneys of Sall1 EGFP/EGFP chimeric neonatal mice were red throughout, indicating that the kidneys were entirely composed of DsRed-marked ESC-derived cells (Sall1 EGFP/EGFP in Figure 1B), and were of normal size. The brains of Sall1 1 EGFP/EGFP chimeric mice contained both EGFP-expressing and DsRed-negative 

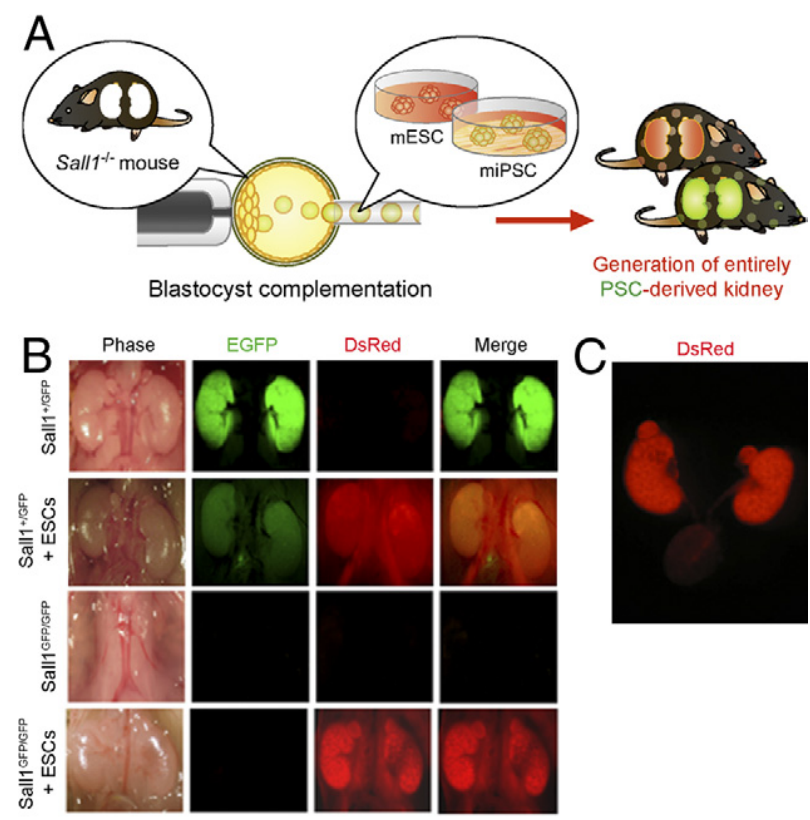

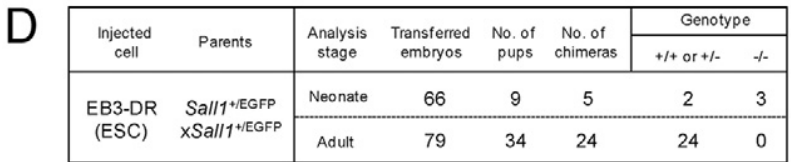

Figure 1. ESC-derived kidneys generated by blastocyst complementation. A: Scheme for the experimental design for PSC-derived kidney generation. Embryos obtained from Sall1 knock-out mice for kidney generation are injected with ESCs (EB3-DsRed) or iPSCs (GT3.2). Genotyping by PCR is performed; mice with entirely ESC- or iPSC-derived kidneys are generated. B: To identify kidneys clearly as being ESC derived or host derived, embryos obtained from intercrosses of Sall1 EGFP knock-in mice (Sall1 ${ }^{+ \text {EGFP }}$ ) are injected with DsRed-marked ESCs. The kidneys of Sall1 ${ }^{\mathrm{EGFP} / \mathrm{EGFP}}$ mice complemented with ESCs are entirely positive for DsRed, indicating their ESC origin (Sall1 ${ }^{\mathrm{EGFP} / \mathrm{EGFP}}+$ ESCs in B). The kidneys of Sall1 ${ }^{+/ \mathrm{EGFP}}$ mice complemented with ESCs are positive for both DsRed and EGFP, indicating a mosaic of both ESC-derived and host-derived cells ( Sall1 ${ }^{+/ E G F P}+$ ESCs in B). C: Representative kidney almost entirely composed of DsRed-positive cells. D: Result of embryo manipulation and the genotype of Sall1 mutant.

cells and EGFP-negative and DsRed-expressing cells (Figure 2G). By contrast, the kidney contained EGFPnegative and DsRed-expressing ESC-derived cells, but not EGFP-expressing and DsRed-negative host cells (Figure 2H). In ESC-derived Sall1 EGFP/EGFP chimeric kidneys, host cells of collecting systems that did not express Sall1 were included in the EGFP-negative and DsRednegative fraction (Figure $2 \mathrm{H}$ ). These results indicate that ESC-derived kidney was formed in Sall1 ${ }^{\text {EGFP/EGFP mice }}$ by blastocyst complementation. A photograph of isolated kidneys, ureters, and bladder demonstrates the different densities of ESC-derived cells; although the kidneys were derived entirely from ESCs, the ESC contribution to ureters and bladder was minimal (Figure 1C). As expected, in both Sal/1 EGFP/EGFP and Sall/1/EGFP mice injected with DsRed-ESCs, ESC-derived cells contributed to all of the non-kidney tissues of the body, including adrenal gland, ureter, bladder, muscle, and adipose tissue (data not shown). The extent of the contribution varied from tissue to tissue and also from mouse to mouse depending on the individual degree of chimerism. Retrospective genotyping in a small population revealed that mice with
Sall $1^{\text {EGFP/EGFP }}$, Sall $1^{+/ E G F P}$, and Sall $1^{+/+}$genotypes were born with Mendelian frequency (Figure 1D). These macroscopic and flow cytometry results substantiated that kidney had been formed in Sall1 ${ }^{\text {EGFP/EGFP }}$ mice via blastocyst complementation. However, no complemented mouse pup survived to adulthood (Figure 1D). By contrast, ESC-derived Sall $1^{+/ E G F P}$ and Sall $1^{+/+}$chimeric mice grew into adulthood (Figure 3, A and B). Moreover, ESCderived Sall $1^{+/ E G F P}$ chimeric mice and Sall $1^{+/+}$chimeric mice were fertile (Figure 3C). An ESC-marking gene,

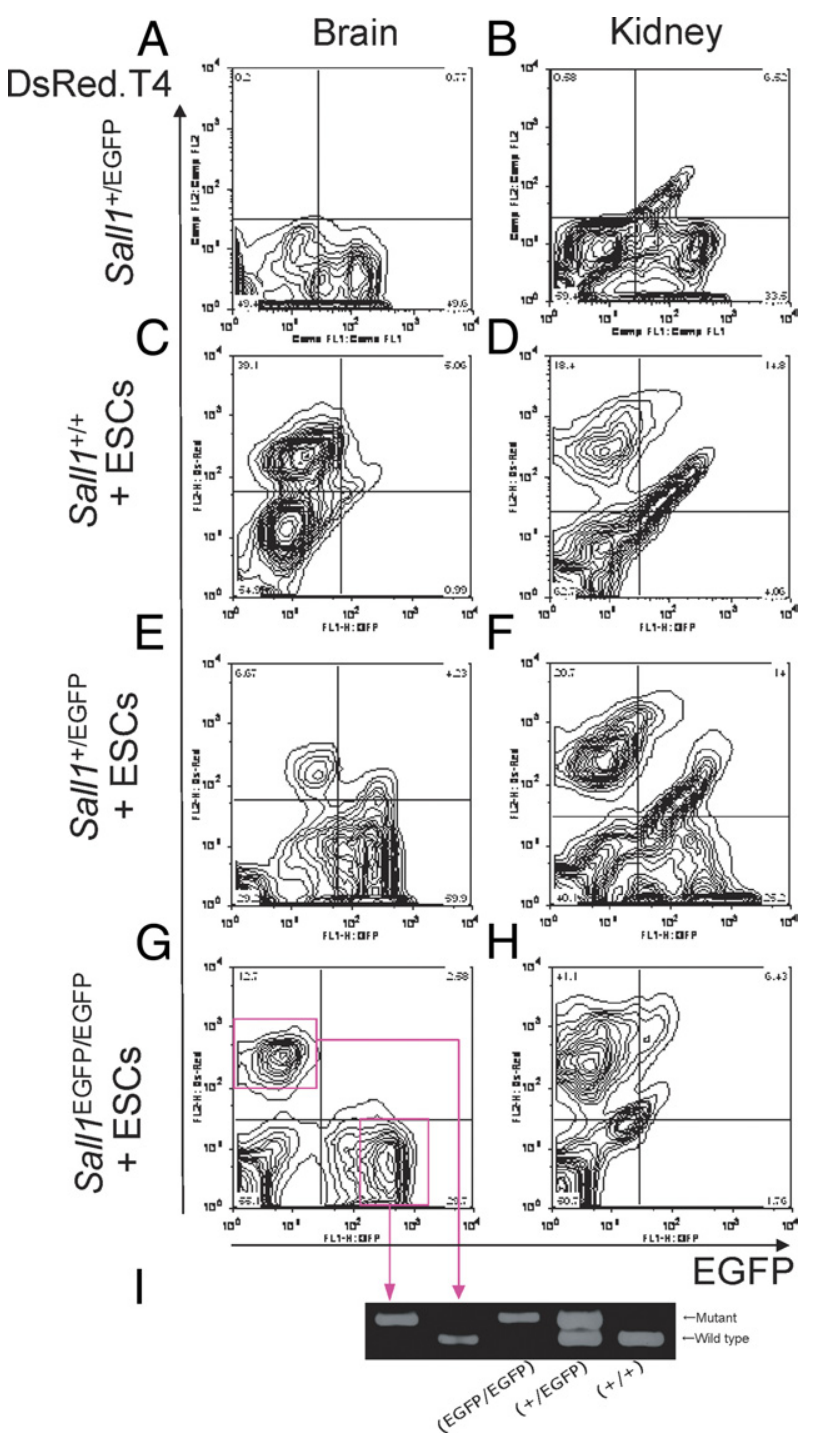

Figure 2. Flow cytometry analysis and genotyping in ESC-derived brains and kidneys. In Sall1 ${ }^{+/ E G F P}$ mice without ESC-chimerism, the brain and kidneys (A and $\mathbf{B}$, respectively) contained EGFP-expressing and DsRednegative cells. Moreover, the kidneys contained a few EGFP-expressing and DsRed-expressing cells that showed autofluorescence. In Sall1 ${ }^{+/+}$ chimeric mice, the brain and kidneys (C and $\mathbf{D}$, respectively) contained EGFP-negative \&DsRed-expressing cells that were derived from ESCs. In Sall1 ${ }^{+/ E G F P}$ chimeric mice, the brain and kidneys (E and $\mathbf{F}$, respectively) contained both EGFP-expressing and DsRed-negative host cells and EGFP-negative and DsRed-expressing ESC-derived cells. G: In Sall1 ${ }^{\text {EGFP/ }}$ EGFP chimeric mice, the brain contained both EGFP-expressing and DsRed-negative host cells and EGFP-negative and DsRed-expressing ESCderived cells. H: Kidneys contained EGFP-negative and DsRed-expressing ESC-derived cells, but not EGFP-expressing and DsRed-negative cells. I: Genotyping PCR was performed on each fraction of sorted cells, as shown by boxed areas in $\mathbf{G}$. 

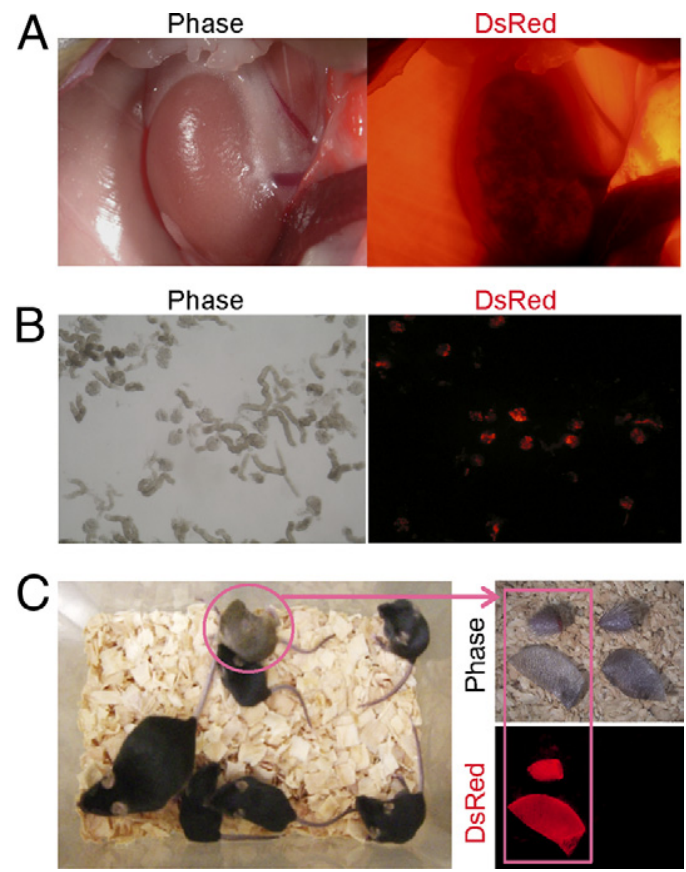

Figure 3. ESC-derived Sall1 $1^{+/ E G F P}$ chimeric mice in adulthood. A: Kidney, muscle, and adipose tissue contained various contributions of ESC-derived cells. B: In ESC-derived chimeric kidneys fragmented with sieve mesh, some glomeruli and some tubules consisted of both ESC-derived cells and hostderived cells. C: ESC-derived Sall1 ${ }^{+/ E G F P}$ chimeric mice were fertile. An ESC-marking gene, DsRed, was transmitted to progeny whose organs all exhibited DsRed fluorescence (circle in left panel, square in right panel)

DsRed, was transmitted to progeny whose organs all exhibited DsRed fluorescence. Figure 4 shows representative sections (H\&E and PAM staining) of ESC-derived kidneys from pups of complemented Sall1 EGFP/EGFP hosts. The glomerular capillary loops and basement membrane showed normal development, with blood cells in the glomerular capillary lumina (Figure 4, B and C). The glomerular podocytes and epithelium of Bowman's capsule were distinct, with open periglomerular urinary spaces (consistent with urine production). That is, the kidneys of these mice were grossly and histologically normal. Moreover, we examined these kidney tissues further for contributions of donor ESCs to different kidney lineages. On immunohistochemical study of kidneys of pups developed from embryos complemented with Sall1 EGFP/EGFP blastomeres, the nephron epithelia (including the condensed mesenchyme, pretubular aggregate, comma-shaped bodies, s-shaped bodies, glomerular podocytes, Bowman's epithelia, proximal tubules, and distal tubules) and the renal stroma (including the cortical stroma and medullary stroma) consisted entirely of DsRed-positive cells (podocytes: Figure 4F; proximal tubules: Figure 4G; other data not shown). By contrast, both DsRed-positive cells and DsRed-negative cells were seen in collecting ducts (Figure 4H). Therefore, the tissues seen in this case were of ESC origin, with the exception of those in collecting-tubule epithelia. These data strongly indicate that we could generate ESC-derived kidneys by blastocyst complementation.

\section{Generation of Kidney from iPSCS}

We next addressed whether we could generate kidney from iPSCs instead of from ESCs. We used blastocysts derived from another Sall1 knock-out mice, in which EGFP was not inserted, as injected iPSCs were marked with EGFP (Figure 1A). As expected, on macroscopy the kidneys of Sall $1^{+/-}$or Sall $1^{+/+}$chimeric mice were a mixture of host-derived cells and EGFP-marked iPSC-derived cells, as in whole-body chimerism (Sall $1^{+/-}$in Figure $5 \mathrm{~A}$ ). In contrast, the kidneys of Sall1 ${ }^{-1-}$ chimeric mice were almost entirely composed of EGFP-marked iPSC-derived cells (Sall1 $1^{-1-}$ in Figure 5, A and B). These iPSC-derived kidneys of Sall $1^{-1-}$ chimeric mice were grossly normal in shape and size. As iPSC-derived kidneys had urine-filled bladders, those kidneys were inferred to communicate with ureters and to function well. In previous reports, the presence of bladders full of urine was shown to reflect functionality of the renal and urological systems in newborn mice. ${ }^{6,12}$ Although we expect that the produced
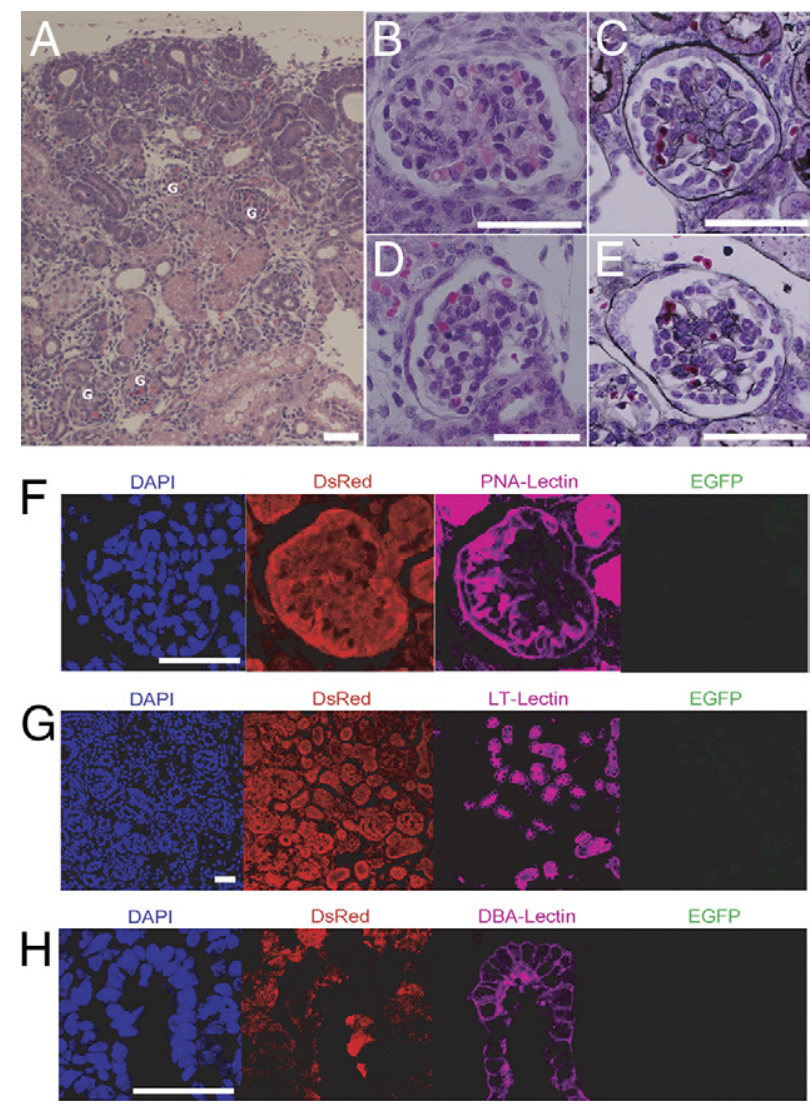

Figure 4. Histology and immunofluorescence of ESC-derived kidneys at the neonatal stage. A: Normal morphology in sections of kidney from Sall1 ${ }^{\text {EGFP/ }}$ EGFP chimera. Mature glomeruli $(G)$ were found. B and C: Glomeruli were normal in appearance in the Sall1 ${ }^{\text {EGFP/EGFP }}$ chimeric kidney. $\mathbf{D}$ and E: Glomerular features were normal in a wild-type 129/Ola strain from which ESCs had been established. H\&E staining: A, B, and D; PAM staining: C, E and $\mathbf{F}-\mathbf{H}$ : Sections were stained with various lectins and anti-DsRed antibody; DAPI was used for nuclear counterstaining. F: PNA-lectin-positive glomerular podocytes were $¥$ DsRed positive and EGFP negative. G: LT-lectin-positive proximal tubular epithelium was DsRed positive and EGFP negative. Most of the LT-lectin-negative tubular epithelia, including that of the distal tubules, were also DsRed-positive and EGFP-negative. H: DBA-lectin-positive collecting tubule epithelia were composed of both DsRed-positive ESC-derived cells and DsRed-negative host cells. Scale bar $=50 \mu \mathrm{m}$. 


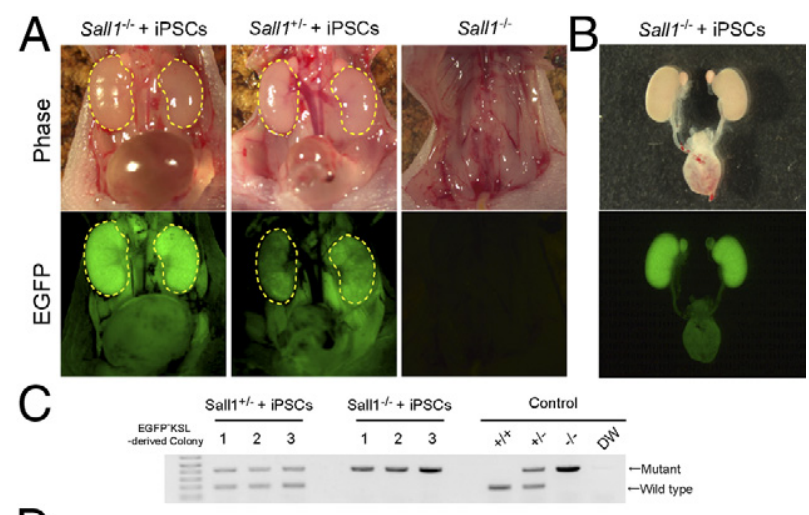

D

\begin{tabular}{|c|c|c|c|c|c|c|c|}
\hline \multirow{2}{*}{$\begin{array}{l}\text { Injected } \\
\text { cell }\end{array}$} & \multirow[b]{2}{*}{ Parents } & \multirow{2}{*}{$\begin{array}{c}\text { Analysis } \\
\text { stage }\end{array}$} & \multirow{2}{*}{$\begin{array}{l}\text { Transferred } \\
\text { embryos }\end{array}$} & \multirow{2}{*}{$\begin{array}{l}\text { No. of } \\
\text { pups }\end{array}$} & \multirow{2}{*}{$\begin{array}{l}\text { No. of } \\
\text { chimeras }\end{array}$} & \multicolumn{2}{|c|}{ Genotype } \\
\hline & & & & & & $+/+$ or $+/$ & $\%$ \\
\hline $\begin{array}{l}\text { GT3.2 } \\
\text { (iPSC) }\end{array}$ & $\begin{array}{l}\text { Sall } 11^{+k} \\
\times \text { SSall } 1^{+k}\end{array}$ & Neonate & 138 & 37 & 34 & 29 & 5 \\
\hline
\end{tabular}

$\mathrm{E}$

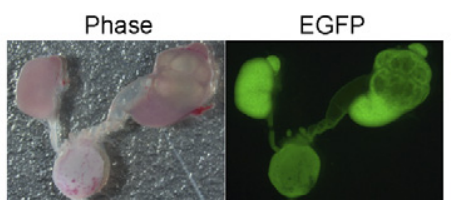

Figure 5. iPSC-derived kidneys generated by blastocyst complementation. A: iPSC-derived kidneys at the neonatal stage. In Sall1 ${ }^{-1-}$ mice complemented with iPSCs, the kidneys were normal in gross appearance and almost entirely positive for EGFP, indicating that they were of iPSC origin (left panels). The bladders of these mice were filled with urine. The kidneys in Sall $^{+/-}$mice complemented with iPSCs were only partially positive for EGFP, indicating the presence of a mosaic of both iPSC-derived and hostderived cells (middle panels). The absence of kidneys is shown in a non-chimeric Sall1 ${ }^{-/-}$mouse at the same developmental stage (right panels). B: A representative kidney is almost entirely composed of EGFPexpressing cells. All of the non-kidney organs were mosaics of cells exhibiting fluorescence and of cells failing to fluoresce. C: Detection of Sall1 mutant genotyping using genomic DNA extracted from colony-forming cells derived after single-cell sorting of EGFP-KSL cells from neonatal or postnatal bone marrow. D: Results of embryo manipulation and genotyping of Sall 1 mutant. E: In one Sall1 ${ }^{-/-}$mouse complemented with iPSCs, the left kidney was hydronephrotic.

urine in newborns is more dilute than that in adults, we were unable to measure its osmolarity because of technical problems. As expected, in both Sall1-1- and Sall $1^{+/-}$mice injected with EGFP-iPSCs, iPSC-derived cells contributed to all of the non-kidney tissues of the body, including adrenal gland, ureter, bladder, muscle, and adipose tissue (data not shown). The extent of the contribution varied from tissue to tissue and also from mouse to mouse depending on the individual degree of chimerism. Single HSC colony assays yielded material for genotyping PCR (Figure 5C). Retrospective genotyping revealed that mice with Sall1 ${ }^{+/+}$, Sall $1^{+/-}$, and Sall $1^{-l_{-}}$ genotypes were born with Mendelian frequency (Figure 5D). In all Sall ${ }^{-1-}$ mice examined, regardless of donor chimerism in other tissues such as peripheral blood (range, $29.1 \%$ to $92 \%$ ), kidneys formed bilaterally. In one mouse, one iPSC-derived kidney was normal and the other was hydronephrotic (Figure 5E). Whether this outcome was due to incomplete iPSC complementation or to other causes (eg, abnormally functioning iPSC-derived cells, Sall $1^{-1-}$ status) remains unclear. These gross findings suggest that iPSC-derived kidneys developed in Sall $1^{-1-}$ mice as a result of blastocyst complementation.
Next, we assessed histological findings in iPSC-derived kidneys. Figure 6 illustrates representative sections (H\&E and PAM staining) of the kidney in pups of complemented Sall $1^{-1-}$ and Sall $1^{+1-}$ host blastocysts. On light microscopy of tissues from complemented Sall $1^{-1-}$ mice, like those from complemented Sall $1^{+/-}$mice, the architecture of renal cortex and medulla was normal (Figure 6A). On immunofluorescence microscopy at low magnification, almost all tissues excluding DB-lectinpositive ureteric buds were EGFP positive. For signal amplification, we assessed the fluorescence of a chromophore coupled with anti-EGFP antibody. The glomerular capillary loops and basement membrane had formed normally, with blood cells in the glomerular capillary lumina (Figure 6B). The glomerular corpuscles were distinct, with open periglomerular urinary spaces consistent with urine production. On ultrastructural study, the glomerular three-layer architecture in complemented Sall $1^{-1-}$ mice was normal, with epithelial foot processes, basement membrane, and fenestrated capillary-loop endothelium (Figure 6C). At high magnification, slit diaphragms were seen (Figure 6D). The kidneys of these mice were grossly and histologically normal. These results clearly indicate that blastocyst complementation could generate both ESC- and iPSC-derived kidneys.

\section{Polyclonal Origin of Single Glomerulus or Nephron in Mouse Chimeras}

To buttress our conclusion that donor iPSCs contributed to different kidney lineages, we analyzed and compared immunohistochemical profiles. In this process, we incidentally confirmed a developmental phenomenon of chimeric mice. Immunohistochemical study of kidney in pups of Sall $1^{+/-}$or Sall $1^{+/+}$iPSC-derived chimeras found tissues of metanephric mesenchymal lineage, including glomerular podocytes in every glomerulus, to contain both EGFP-positive cells and EGFP-negative cells (Figure 6A, Figure 7A-D). Throughout nephrogenesis, the nephron progenitors and epithelia of metanephric mesenchymal lineage (ie, including the condensed mesenchyme, pretubular aggregate, comma-shaped bodies, s-shaped bodies, mature glomeruli, proximal and distal tubules) were similar. Among glomeruli, 28 had mixed EGFP-positive and EGFP-negative podocytes, two had only EGFP-positive podocytes, and one had only EGFPnegative podocytes. Moreover, other metanephric mesenchymal components (including the epithelia of Bowman's capsule and of the proximal and distal tubules) also showed a mixture of EGFP-expressing and nonEGFP-expressing cells (Figure 7D). In summary, individual metanephric mesenchymal components were composed of cells of polyclonal origin in mouse chimeras.

\section{Immunohistochemical Analysis of iPSC-Derived Kidneys}

We examined these kidney tissues further for contributions of donor iPSCs to different cell lineages. On immunohistochemical study of kidneys in pups from com- 


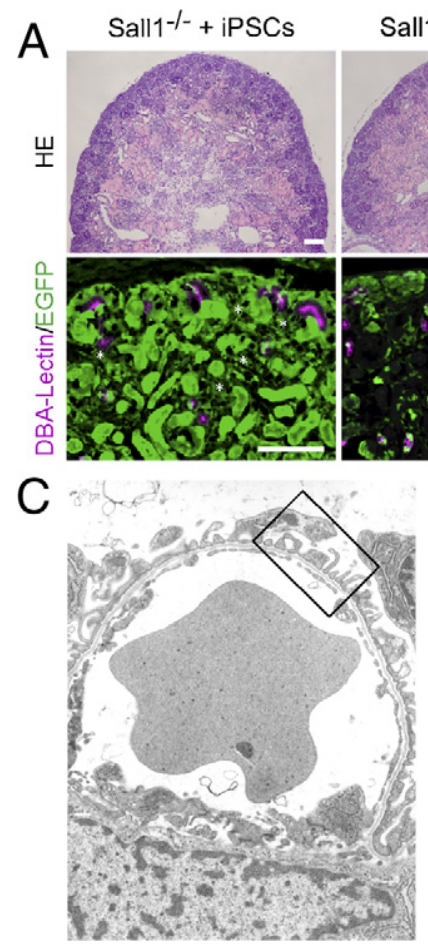

all1 $1^{+/-}+$iPSCs

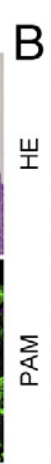

Sall1 $1-+$ iPSCs

Sall $1^{+/-}+$iPSCs

D

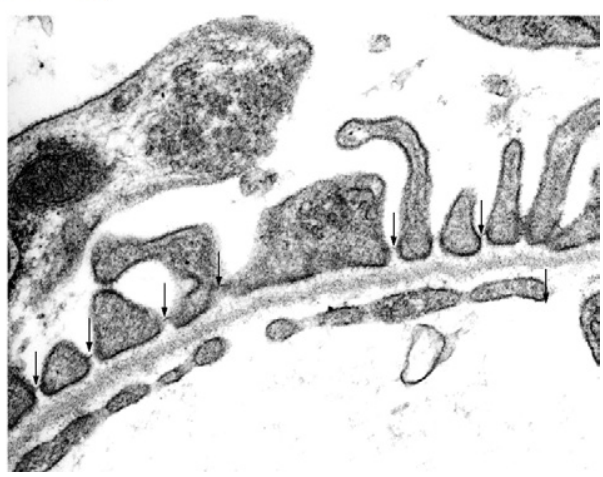

Figure 6. Histology of iPSC-derived kidneys at the neonatal stage. A: Normal morphology in sections of kidney from Sall1 ${ }^{-/-}$chimera. Distribution of iPSCderived cells in sections of iPSC-derived kidney from Sall1 ${ }^{-/-}$and Sall1 ${ }^{+/-}$hosts. Upper panels: Paraffin sections stained with H\&E. Lower panels: Frozen sections stained for DBA-lectin and EGFP. Most of the nephron epithelia were of iPSC origin, but this was not the case for the DBA-lectin-positive ureteric bud epithelia. The cortical stroma was of iPSC origin (asterisk). Scale bar $=100 \mu \mathrm{m}$. B: Glomeruli were normal in appearance in Sall1 ${ }^{-/-}$and Sall1 ${ }^{+/-}$chimeric kidneys. Sections were stained with H\&E or with PAM technique. Scale bar $=50 \mu \mathrm{m}$. C: Transmission electron micrograph of glomerulus in iPSCderived kidney. The triple-layer architecture of the glomerular capillary in complemented Sall1 ${ }^{-1}$ mice was normal, showing epithelial foot processes, basement membrane, and fenestrated capillary-loop endothelium. Original magnification, $\times 6000$. D: High-power view of boxed region in $\mathbf{C}$. Foot processes and slit diaphragms (arrows) exhibit normal development. Original magnification, $\times 20,000$. plemented Sall1-1- host blastocysts, the cells of metanephric mesenchymal lineage (including the condensed mesenchyme, pretubular aggregate, commashaped bodies, s-shaped bodies, mature glomeruli, proximal and distal tubules) were of iPSC origin, with the exception of the collecting duct epithelium (Figure 6A, Figure $7 \mathrm{E}-\mathrm{G}$ ). Most of the renal stroma (including the cortical stroma, medullary stroma, and kidney capsule) was of iPSC origin (Figure 6A, Figure $8 \mathrm{~A}-\mathrm{C}$ ). This was to be expected, as the collecting ducts and ureters are originally derived from the ureteric bud; that is, development of these structures did not occur under the direct influence of Sall1.

The cortical stroma $(A)$, in which nephron epithelia lie, medullary stroma (B), and Liv2-expressing kidney capsule (C) were replaced by EGFP-positive iPSC-derived cells. With respect to vascular systems, all of the endothelia of vascular elements (including the arteries, arterioles, glomerular endothelia, peritubular capillaries, and venules)

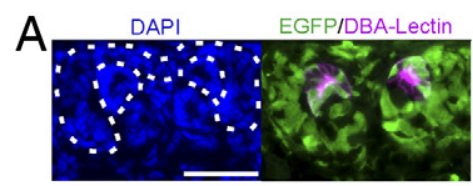

B

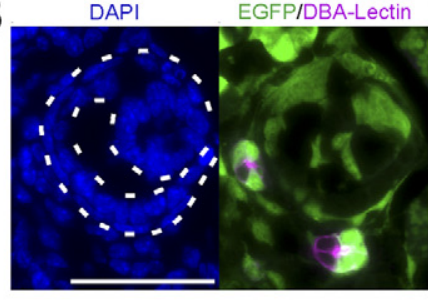

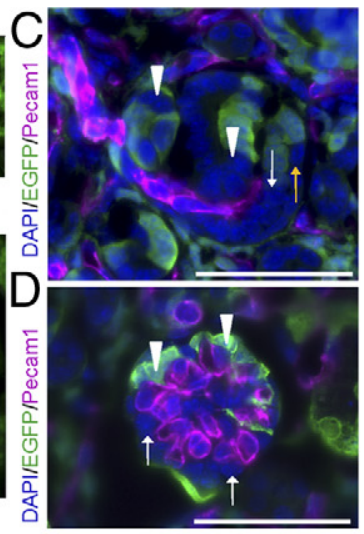

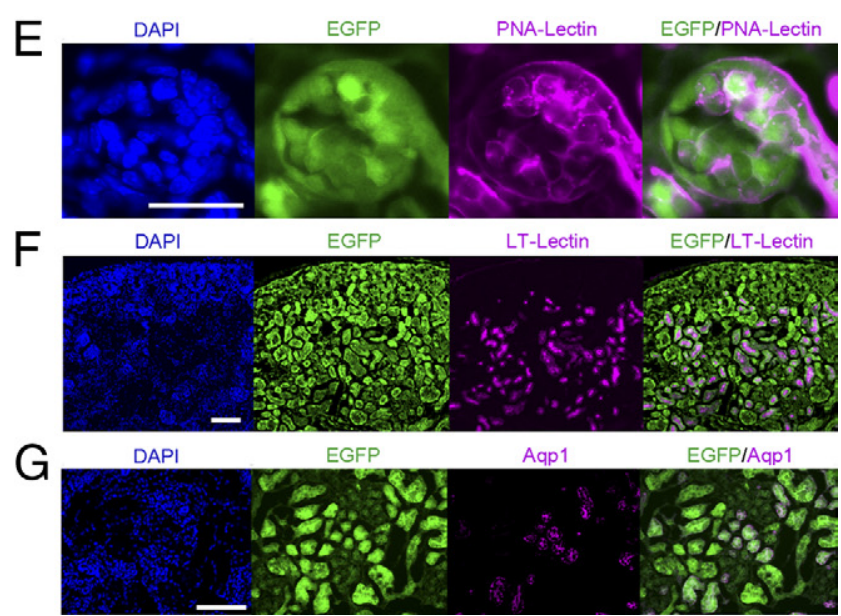

Figure 7. Immunofluorescence-microscopy studies of tissues of metanephric mesenchymal lineages in iPSC-derived kidneys at the neonatal stage. Polyclonal origin of a single glomerulus or tubule in mouse chimeras (using iPSC-derived kidney of Sall1 ${ }^{+/-}$chimeras). A and B: Immunofluorescence in the iPSC-derived kidney of Sall1 ${ }^{+/-}$chimeras. Metanephric mesenchymal lineages including condensed mesenchyme (dashed line in A), and comma-shaped bodies (dashed line in B) were mosaics of both iPSC- and host-derived cells. C: In the s-shaped body stage of the Sall1 ${ }^{+/-}$chimeric kidney, a glomerular crevice with Pecam1-positive endothelial cells was seen. Presumptive glomerular podocytes (white arrow), Bowman's epithelia (yellow arrow), and the upper portion of the s-shaped body (arrowhead) were mosaics of both iPSC- and host-derived cells. D: In the mature glomerulus of Sall1 ${ }^{+/-}$chimeric kidney, glomerular podocytes were hybrids of both EGFP-positive cells (arrowheads) and EGFP-negative cells (arrows). Scale bar $=50 \mu \mathrm{m}$. Immunofluorescence, iPSC-complemented Sall1 ${ }^{-}-$ kidney (E-G). E: PNA-lectin-positive glomerular podocytes were totally composed of EGFP-positive iPSCs. F: LT-lectin-positive proximal tubular epithelia were of iPSC origin. G: In the medulla of the iPSC-complemented Sall1 ${ }^{-1-}$ kidney, tubular epithelia of Aqp1-positive thin limbs of loops of Henle were of iPSC origin. Scale bars: 50 $\mu \mathrm{m}(\mathbf{A}-\mathbf{D}) ; 25 \mu \mathrm{m}(\mathbf{E}) ; 100 \mu \mathrm{m}(\mathbf{F}$ and $\mathbf{G})$. 

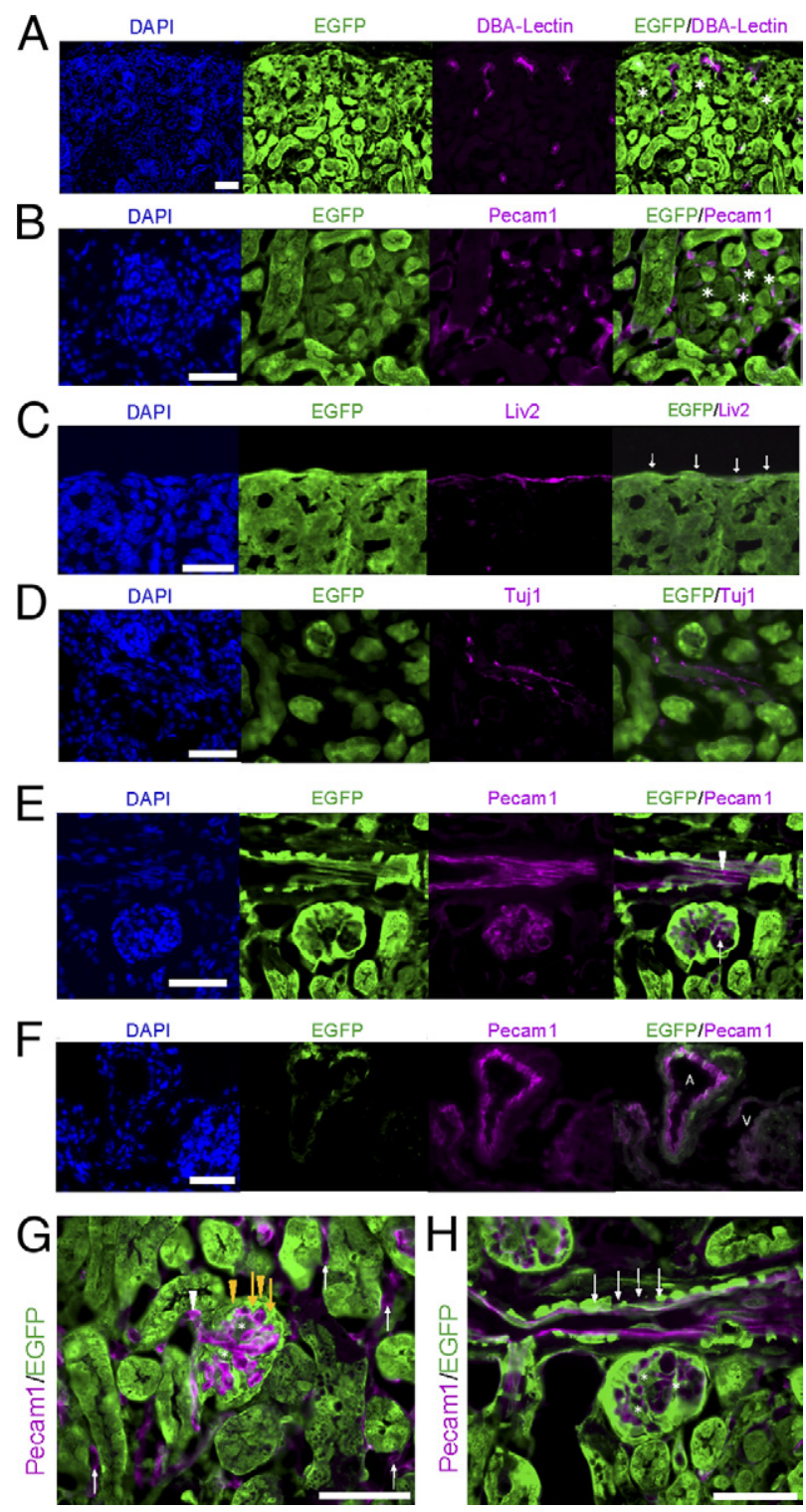

Figure 8. Immunofluorescence-microscopy studies of stromal components and vasculature in iPSC-complemented Sall1 ${ }^{-/-}$kidneys at the neonatal stage. A: Most of the cortical stroma (asterisk) in which nephron epithelia lie was replaced by EGFP-positive iPSC-derived cells. B: The medullary stroma (asterisk) was of iPSC origin. C: Liv2-expressing kidney capsule, a layer of epithelial cells (arrows) cloaking the cortical surface, was of iPSC origin. D: Tuj1-expressing nerves around vessels in iPSC-derived kidney were not composed of iPSCs. E: Pecam1-expressing endothelium of glomerulus (arrow) and of interlobular artery (arrowhead) was chimeric, as shown by EGFP fluorescence. F: The Pecam1-expressing endothelium of segmental renal artery (A) and vein (V) was chimeric for EGFP. G: Pecam1-expressing endothelium of glomerulus, arteriole (white arrowhead), and peritubular capillary (white arrows) was chimeric for EGFP. Glomerular podocytes (yellow arrows) and Bowman's capsule epithelium (yellow arrowheads) were composed of EGFP-positive iPSC-derived cells. The glomerular mesangium (asterisk) was also of iPSC origin. H: In a section parallel to material shown in $\mathbf{E}$, glomerular mesangium (asterisk) was of iPSC origin. Leiomyocytes (arrows) of interlobular artery were chimeric for EGFP. Scale bar $=50$ $\mu \mathrm{m}$.

showed chimeric organization (Figure 8E-H). The glomerular mesangium, but not the leiomyocytes of vessel walls, was of iPSC origin (Figure 8, G and $\mathrm{H}$ ). In addition, nearly all juxtavascular or perivascular nerves were not of iPSC origin (Figure 8D). These results indicate that in all mice, although
iPSCs supplied all kidney cell lineages, collecting duct epithelia and kidney stromal elements such as vessels and nerves were composites of host- and iPSC-derived cells.

\section{Discussion}

We have taken an innovative approach, that of blastocyst complementation, to organ regeneration and have demonstrated that this technique can be used to generate donor PSCs-derived functional kidney. If an empty developmental niche for an organ is provided (as with the $P d \times 1^{-1-}$ mouse and the pancreatic niche ${ }^{5}$ ), PSCs-derived cellular progeny can occupy that niche and developmentally produce a solid organ in the vacant space. Several groups have used the same technique to study development of thymic epithelia ${ }^{13}$ and compensation for cardiac defects, ${ }^{14}$ to determine clonal origins in yolk sac hematopoiesis and germ cell development, ${ }^{15,16}$ or to define developmental organ sizes of liver and pancreas. ${ }^{17}$ Here, we applied this technique to the generation of kidneys.

Our present findings demonstrated that epithelial cellular lineages originating from the metanephric mesenchyme were entirely replaced by the progeny of injected PSCs in Sall1 ${ }^{-1-}$ chimeric mice (Figure 4, Figure 7). Sall1 is expressed in the metanephric mesenchyme-derived structures in the developing kidney, and is essential not only for the initial interactions between the mesenchyme and the ureteric buds but also for the growth and development of the metanephric mesenchyme after those interactions. Indeed, in vitro assays indicated that the Sall1-expressing cell population in the metanephric mesenchyme contains multipotent nephron progenitors, and that colony formation after plating was impaired in EGFP-positive cells from Sall1 EGFP/EGFP mouse kidneys. ${ }^{18}$ During nephrogenesis in the setting of blastocyst complementation, host embryo-derived cells in Sall1-deficient mice could not compete with injected normal ESC- or iPSC-derived cells. In addition, all renal stromal elements (ie, cortical stroma, medullary stroma, and kidney capsule) were composed of PSC-derived cells, suggesting that these tissues' development also depends on Sall1 expression. It is worth noting that glomerular mesangial cells, but not leiomyocytes of vessel walls, were entirely composed of PSC-derived cells, although the cellular phenotype of mesangial cells reportedly resembles that of vascular leiomyocytes. ${ }^{19}$ Our data therefore strongly indicate that Sall1 exerts cell-autonomous functions in various lineages, including that of the metanephric mesenchyme, and that Sall1 mutant mice could be useful as harboring a developmental niche in which to regenerate the major parts of the kidney by blastocyst complementation.

We consider that the mechanism for the rescue of tissues of renal stromal lineage including the renal capsule was related to cell-autonomous function during organ development. Sall1 expression is reported not only in metanephric mesenchyme but also in Foxd1-expressing stromal components, although in the latter Sall1 expression is relatively weak. ${ }^{18}$ Our finding that the stroma is 
also replaced by PSC descendants may suggest that Sall1 is also required for this lineage cell-autonomously, although lineage-specific Sall1 deletion would be needed to test this hypothesis.

By contrast, collecting ducts derived from ureteric buds, microvascular endothelial cells including the glomerular endothelia, and leiomyocytes were not entirely composed of PSC-derived cells (Figure 8). These findings are consistent with the fact that Sall1 is not expressed in the renal lineages mentioned above at kidney development. Therefore mutant mice lacking these lineages should be combined with Sall1 knock-out mice if kidneys wholly composed of PSC descendants are to be generated.

In this study, none of the mouse pups with complemented kidneys survived to adulthood. Some chimeric mouse models with unexpectedly lethal phenotypes have been reported. ${ }^{20,21}$ This suggests that chimerism does not always rescue mice from embryonic lethality, perhaps partly due to the insufficient multipotency of ESCs or to low proportional chimerism. Sall $1^{-1-}$ mice not only exhibited renal agenesis but also nursed poorly, as inferred from the absence of intragastric milk visible through the abdominal wall. ${ }^{6}$ This was also true for blastocyst-complemented Sall $1^{-1-}$ pups. Because Sall1 is expressed in the brain, it may mediate the development of nerve pathways necessary for suckling function. Four Sall family genes were previously identified, Sall1 to Sall4, and were demonstrated to act synergetically as transcription factors. ${ }^{22,23}$ Therefore, each Sall family is possible to resemble in cellular function, and the phenotypes of the mutant mice may be similar each other. Among them, Sall1 expression co-localizes with other Sall family expression, at sites such as the brain, kidney, limb buds, and heart. Indeed, Sall3 mutant mice show anatomical defects in the brain, ${ }^{24}$ permitting the inference that Sall1-deficient mice, without such defects, may have neurological functional abnormalities. Moreover, suckling ability can be adversely affected by other causes, including seeking the nipple, recognizing the nipple, orally grasping the nipple, sucking, and transport of oral contents to the back of the mouth, with swallowing. As expected, in PSC-injected Sall $1^{-1-}$ mice, both PSC-derived cells and host cells contributed to all non-kidney tissues. Therefore, neuromuscular and endocrine organs necessary for proper suckling were composites of both cell types, and physiological function may have been imperfectly recovered in these organs despite PSC-derived complementation. This perhaps illustrates an important aspect of blastocyst complementation, in that exogenous PSC-derived cells may not compensate for cell-intrinsic developmental defects that are not occasioned by cell loss. Alternatively, chimerism achieved by exogenous cells may not be sufficient to compensate fully for Sall1 deficiency at some sites, and functional defects caused by Sall1 deficiency may persist. Because of the lack of progression to adulthood, it was not possible to observe functioning of PSC-derived kidneys in adult mice. However, the observation of urine accumulation in the bladders of neonatal Sall $11^{-1-}$ mice derived from iPSC-complemented blastocysts strongly suggests that iPSC-derived kidneys were functional.

Previously we had successfully generated rat pancreas in mouse by injecting rat iPSCs into $P d \times 1^{-1-}$ mouse embryos. ${ }^{5}$ In this study, we also injected rat iPSCs into Sall $1^{-1-}$ mouse blastocysts; however, to date, we have not been able to generate rat kidneys in mice (unpublished observations). If the key molecules in mice involved in the interactions of the mesenchyme and the ureteric buds do not cross-react with those in rats, to vault this hurdle it would be necessary to generate a host mouse strain lacking all of the lineages that contribute to the kidney.

In summary, we successfully generated kidneys from PSCs via blastocyst complementation. This accomplishment may open doors to insights into the cellular and molecular mechanisms of kidney development.

\section{Acknowledgments}

We thank Satoshi Yamazaki, Sanae Hamanaka, Yuji Yamazaki, Yohei Morita, Hiroko Tsukui, and Tsutomu Kohda for their technical advice and Lin Franklin for critical review of the manuscript.

\section{References}

1. Summit SCotl: Organ trafficking and transplant tourism and commercialism: the Declaration of Istanbul. Lancet 2008, 372:5-6

2. Takahashi K, Yamanaka S: Induction of pluripotent stem cells from mouse embryonic and adult fibroblast cultures by defined factors. Cell 2006, 126:663-676

3. Takahashi K, Tanabe K, Ohnuki M, Narita M, Ichisaka T, Tomoda K, Yamanaka S: Induction of pluripotent stem cells from adult human fibroblasts by defined factors. Cell 2007, 131:861-872

4. Chen J, Lansford R, Stewart V, Young F, Alt FW: RAG-2-deficient blastocyst complementation: an assay of gene function in lymphocyte development. Proc Natl Acad Sci USA 1993, 90:4528-4532

5. Kobayashi T, Yamaguchi T, Hamanaka S, Kato-Itoh M, Yamazaki Y, Ibata M, Sato H, Lee YS, Usui J, Knisely AS, Hirabayashi M, Nakauchi $\mathrm{H}$ : Generation of rat pancreas in mouse by interspecific blastocyst injection of pluripotent stem cells. Cell 2010, 142:787-799

6. Nishinakamura R, Matsumoto $Y$, Nakao K, Nakamura K, Sato A, Copeland NG, Gilbert DJ, Jenkins NA, Scully S, Lacey DL, Katsuki M, Asashima M, Yokota T: Murine homolog of SALL1 is essential for ureteric bud invasion in kidney development. Development 2001, 128:3105-3115

7. Takasato M, Osafune K, Matsumoto Y, Kataoka Y, Yoshida N, Meguro $H$, Aburatani $H$, Asashima M, Nishinakamura R: Identification of kidney mesenchymal genes by a combination of microarray analysis and Sall1-GFP knockin mice. Mech Dev 2004, 121:547-557

8. Hooper M, Hardy K, Handyside A, Hunter S, Monk M: HPRT-deficient (Lesch-Nyhan) mouse embryos derived from germLine colonization by cultured cells. Nature 1987, 326:292-295

9. Niwa H, Miyazaki J, Smith AG: Quantitative expression of Oct-3/4 defines differentiation, dedifferentiation or self-renewal of ES cells. Nat Genet 2000, 24:372-376

10. Okabe M, Otsu M, Ahn DH, Kobayashi T, Morita $Y$, Wakiyama $Y$, Onodera M, Eto K, Ema H, Nakauchi $\mathrm{H}$ : Definitive proof for direct reprogramming of hematopoietic cells to pluripotency. Blood 2009, 114:1764-1767

11. Nagy A, Gertsenstein M, Vintersten K, Behringer R: Manipulating the mouse embryo: a laboratory manual. Cold Spring Harbor, NY, Cold Spring Harbor Laboratory Press, 2003

12. Hammes A, Guo JK, Lutsch G, Leheste JR, Landrock D, Ziegler U, Gubler MC, Schedl A: Two splice variants of the Wilms' tumor 1 gene 
have distinct functions during sex determination and nephron formation. Cell 2001, 106:319-329

13. Muller SM, Terszowski G, Blum C, Haller C, Anquez V, Kuschert S, Carmeliet P, Augustin HG, Rodewald HR: Gene targeting of VEGF-A in thymus epithelium disrupts thymus blood vessel architecture. Proc Natl Acad Sci U S A 2005, 102:10587-10592

14. Fraidenraich D, Stillwell E, Romero E, Wilkes D, Manova K, Basson CT, Benezra R: Rescue of cardiac defects in id knockout embryos by injection of embryonic stem cells. Science 2004, 306:247-252

15. Ueno H, Weissman IL: Clonal analysis of mouse development reveals a polyclonal origin for yolk sac blood islands. Dev Cell 2006, 11:519-533

16. Ueno H, Turnbull BB, Weissman IL: Two-step oligoclonal development of male germ cells. Proc Natl Acad Sci USA 2009, 106:175-180

17. Stanger BZ, Tanaka AJ, Melton DA: Organ size is limited by the number of embryonic progenitor cells in the pancreas but not the liver. Nature 2007, 445:886-891

18. Osafune K, Takasato M, Kispert A, Asashima M, Nishinakamura R: Identification of multipotent progenitors in the embryonic mouse kidney by a novel colony-forming assay. Development 2006, 133:151-161

19. Lindahl P, Hellstrom M, Kalen M, Karlsson L, Pekny M, Pekna M, Soriano P, Betsholtz C: Paracrine PDGF-B/PDGF-Rbeta signaling controls mesangial cell development in kidney glomeruli. Development 1998, 125:3313-3322
20. Scacheri PC, Crabtree JS, Novotny EA, Garrett-Beal L, Chen A, Edgemon KA, Marx SJ, Spiegel AM, Chandrasekharappa SC, Collins FS: Bidirectional transcriptional activity of PGK-neomycin and unexpected embryonic lethality in heterozygote chimeric knockout mice. Genesis 2001, 30:259-263

21. He Y, Hakvoort TB, Vermeulen JL, Lamers WH, Van Roon MA: Glutamine synthetase is essential in early mouse embryogenesis, Dev Dynam 2007, 236:1865-1875

22. Sakaki-Yumoto M, Kobayashi C, Sato A, Fujimura S, Matsumoto $Y$, Takasato M, Kodama T, Aburatani H, Asashima M, Yoshida N, Nishinakamura R: The murine homolog of SALL4, a causative gene in Okihiro syndrome, is essential for embryonic stem cell proliferation, and cooperates with Sall1 in anorectal, heart, brain and kidney development. Development 2006, 133:3005-3013

23. Kawakami $Y$, Uchiyama Y, Rodriguez Esteban C, Inenaga T, KoyanoNakagawa N, Kawakami H, Marti M, Kmita M, Monaghan-Nichols P, Nishinakamura R, Izpisua Belmonte JC: Sall genes regulate regionspecific morphogenesis in the mouse limb by modulating Hox activities. Development 2009, 136:585-594

24. Parrish M, Ott T, Lance-Jones C, Schuetz G, Schwaeger-Nickolenko A, Monaghan AP: Loss of the Sall3 gene leads to palate deficiency, abnormalities in cranial nerves, and perinatal lethality. Mol Cell Biol 2004, 24:7102-7112 